\title{
Differentiating Defect and Basal Plane Contributions to the Surface Energy of Graphite Using Inverse Gas Chromatography
}

Auren Ferguson, Ivan T Caffrey, Claudia Backes, Jonathan N Coleman* and Shane D Bergin* School of Physics, CRANN and AMBER Research Centres, Trinity College Dublin, Ireland *berginsd@tcd.ie, colemaj@tcd.ie

ABSTRACT: Historically, reported values for the surface energy of graphite have covered a very wide range. Here we use finite-dilution inverse gas chromatography (FD-IGC) to show that the dispersive component of the surface energy of graphite has contributions from edge and basal plane defects as well as from the hexagonal carbon lattice. The surface energy associated with the defect-free hexagonal lattice is measured at high probe-coverage to be $63 \pm 7 \mathrm{~mJ} / \mathrm{m}^{2}$, independent of graphite type. However, the surface energy measured at low probe coverage varied from $125-175 \mathrm{~mJ} / \mathrm{m}^{2}$ depending on the graphite type. Simulation of the FDIGC output for different binding site distributions allows us to associate this low-coverage surface energy with the binding of probe molecules to high energy defect sites. Importantly, we find the rate of decay of surface energy with probe coverage to carry information about the defect density. By analysing the dependence of these properties on flake size, it is possible to separate out the contributions of edge and basal plane defects, estimating the basal plane defect content to be $\sim 10^{15} \mathrm{~m}^{-2}$ for all graphite samples. Comparison with simulation gives some insights to the basal plane and defect binding energy distributions.

\section{INTRODUCTION}

Graphite has been studied scientifically for well over a century and is one of the best understood materials known to man. For example, its electronic properties have been studied exhaustively since $1947 .{ }^{1}$ However, one property of graphite which is not so well understood is its surface energy. The reasons for this are twofold. Firstly, a very wide range of values have been reported in the literature for the surface energy of graphite, from $\sim 27-875 \mathrm{~mJ} / \mathrm{m}^{2}{ }^{2,3} \mathrm{~A}$ contributing factor is that graphite from different sources can display different surface energies. Secondly, it is known that graphitic surfaces are extremely energetically heterogeneous. ${ }^{4}$ This means that the surface energy can vary spatially over the surface of a single graphite sample. 
This is unfortunate as a knowledge of a materials surface energy is critical to the development of an understanding of its interactions with other materials. An example can be found in the production of graphene ${ }^{5}$ by exfoliation of graphite. ${ }^{6-8}$ One method in particular, liquid phase exfoliation (LPE), can be used to produce defect-free graphene in a scalable manner. ${ }^{9}$ Theoretical studies have shown that the best solvents for exfoliating graphite to give graphene are those whose surface energy closely match that of graphene. ${ }^{9}$ However without a proper understanding of the surface energies of either graphite or graphene, solvent optimisation remains completely empirical. Although the best solvents ${ }^{8}$ are found to be those with surface energies close to $70 \mathrm{~mJ} / \mathrm{m}^{2}$, it is not clear that this value represents the true surface energy of graphene/graphite.

The surface energy of a material is a measure of the energy required to produce a unit area of surface. Measuring surface energy of solids is important to a variety of applications or industries. For example, in pharmaceuticals accurate knowledge of surface energy is important due to its influence on wet granulation and film coating. ${ }^{10}$ In addition, surface energy measurements can be used to monitor product batch-to-batch variation. ${ }^{11}$ There are several established methods to measure the surface energy of materials. Two widely used methods are contact angle measurements and inverse gas chromatography (IGC). Contact angle is a wettability measurement where droplets of various liquids are deposited on the material's surface and the surface energy is extracted from the angle the droplet edge makes with the surface. In contact angle measurements, a portion of the surface is completely covered with solvent. This means that, for an inhomogeneous surface, a single number related to the average surface energy is returned. However, no information about the surface inhomogeneity is given. Alternatively, IGC has traditionally involved the injection of minute amounts of probe solvent into a column containing the solid sample (this is generally referred to as infinite dilution inverse gas chromatography, ID-IGC). Then, because of the presence of sample-probe interactions, the time taken for the probe to elute through the column is related to the samples surface energy. The main disadvantage of traditional ID-IGC is that, for an inhomogeneous surface, it predominately probes those sites with the largest sample-probe binding energy and so the greatest contribution to the surface energy. As such, it doesn't give a realistic representation of the intrinsic surface energy of a material. ${ }^{12}$

Surface energy measurements of graphite are further complicated by the fact that the material varies greatly depending on where it was mined and the processes used to refine it. In particular, most samples contain flakes with a wide range of sizes. Previous studies on the 
surface energetics of graphite using ID-IGC suggest that surface heterogeneities are partially due to active functional groups and contaminants ${ }^{13}$ all of which are considered defects from the perfect graphitic surface. The population of such defects or other structural imperfections may vary with graphite source. These factors make its heterogeneous nature unsurprising and mean a spread of surface energy values can be expected regardless of the measurement technique. This combination of measurement inconsistency and the heterogeneous nature of graphite itself means that a new approach to study the surface energetics of graphite is required.

In this work we measure surface energy profiles of a range of graphite types using surface coverage dependent IGC. This technique measures the surface energy of a material as a function of the number of probe molecules interacting with the surface (expressed as surface coverage). In all cases, we find high surface energy at low probe coverage. However, the measured surface energy falls off as the coverage is increased. Informed by simulations, we associate the low-coverage, high surface energy values with the interaction of probe molecules with defects while the full-coverage, low surface energy values are linked to interactions with the defect-free basal plane. We find the full-coverage surface energies to be independent of graphite type. Conversely the low-coverage surface energy scales with the flake size, consistent with its dependence on the population of edge defects.

\section{INVERSE GAS CHROMATOGRAPHY THEORY}

IGC is a versatile and robust surface characterisation technique used in a large variety of fields such as pharmaceuticals, ${ }^{10}$ polymers ${ }^{14}$ and mining. ${ }^{15}$ While other surface energy measurement techniques such as contact angle require a smooth flat surface, IGC holds a distinct advantage in that it can be used on powders, flaked materials and films. Although IGC is primarily used for measuring surface energy of solids, ${ }^{16}$ it also has other applications such as measuring specific surface area, ${ }^{17}$ acid-base interactions, ${ }^{16}$ Hildebrand and Hansen solubility parameters ${ }^{18}$ and glass transition temperatures in polymers. ${ }^{19}$

In IGC, known probe solvents are injected into a glass column containing the sample being examined. The concentration of solvent transmitted through the column is then recorded as a function of time in the form of a chromatogram. The adsorption-desorption behaviour of the probe on the solid surface is inferred from the retention time, $t_{r}$, which is the time taken for the probe to elute through the column. The retention time is used to calculate the net retention volume, $V_{N}$ which is a fundamental thermodynamic property of solid-vapour interactions, using the equation: 


$$
V_{N}=\frac{j}{m} F\left(t_{r}-t_{0}\right)\left(\frac{T}{273.15 \mathrm{~K}}\right)
$$

Here $j$ is the James-Martin correction factor, ${ }^{20} m$ is mass of sample in the column, $F$ is the carrier gas flow rate, $t_{0}$ is the dead time (time taken for non-interacting probe to elute through the column) and $T$ is the column temperature. Once $V_{N}$ is known, it can be used to calculate a range of parameters including acid-base interactions, solubility parameters and most commonly surface energy. The surface energy of a material, $\gamma$, consists of two components, the dispersive surface energy, $\left(\gamma_{d}\right)$ and specific surface energy $\left(\gamma_{s}\right)^{17}$. The dispersive surface energy of a material has its basis in London (or dispersion) interactions ${ }^{21}$ while $\gamma_{s}$ is associated with specific interactions between probe and solvent (i.e. polar interactions and hydrogen bonding on the sample surface). ${ }^{22}$ In this work we focused on $\gamma_{d}$ measurements since the measured values are probe-solvent independent while values obtained for $\gamma_{s}$ depend the specific solvents used. In addition, one would expect the dispersive component to dominate the surface energy of graphite. Two widely used methods of calculating $\gamma_{d}$ using IGC are the Schultz $^{23}$ and Dorris-Gray methods. ${ }^{24}$ The Dorris-Gray method was used in this case as it is known to be more accurate at higher operating temperatures. ${ }^{25}$ In the Dorris-Gray method a series of n-alkanes are used as probes to measure the free energy of adsorption, $\Delta G_{p r o b e}$. The dispersive free energy of one methylene group $\left(\Delta G_{C H 2}\right)$ can be calculated from slope of the alkane line by plotting $\Delta G_{\text {probe }}$ versus the carbon number, $n$ of the alkane probe using:

$$
\Delta G_{C_{2}}=-R T \ln \left(\frac{V_{N, n+1}}{V_{N, n}}\right)
$$

where $T$ is the column temperature and $V_{N}$ is the net retention volume calculated by equation 1 . $\Delta G_{C H 2}$ is related to the work of adhesion, $W_{C H 2}$ by:

$$
\Delta G_{\mathrm{CH}_{2}}=-\mathrm{N}_{a} a_{\mathrm{CH}_{2}} W_{\mathrm{CH}_{2}}
$$

For non-polar probes $W_{\mathrm{CH}_{2}}$ is found via the geometric mean approximation using the Fowkes relation: ${ }^{26}$

$$
W_{C H_{2}}=2 \sqrt{\gamma_{d} \gamma_{d}^{C H_{2}}}
$$


where $\gamma_{d}{ }^{\mathrm{CH} 2}$ is the dispersive surface energy of a methylene group which is calculated by $\gamma_{d}{ }^{C H 2}=35.6+0.058(293-T) \cdot{ }^{17}$ By combining equations $2,3,4$ and rearranging we get an expression for $\gamma_{d}$, the dispersive surface energy of the solid sample:

$\gamma_{d}=\frac{1}{4 \gamma_{d}^{C H_{2}}}\left[\frac{R T \ln \left(V_{N, n+1} / V_{N, n}\right)}{N_{a} a_{C H_{2}}}\right]^{2}$

where $R T \ln \left(V_{N, n+1} / V_{N, n}\right)$ is the slope of alkane line from the plot $R T \ln V_{N}$ versus carbon number of the alkane probe.

The experiments described here were performed using a Surface Energy Analyser (SEA). The SEA is the latest generation of IGC apparatus and makes measuring the surface energy of highly energetically heterogeneous materials such as graphite more reliable through the ability to inject precise amounts of probe molecule, corresponding to a specific surface coverage. When very low concentrations of probe are injected into the column, the surface coverage is very low. As a result, only probe-sample interactions occur and only surface sites with high probe-surface binding energy are investigated. This is the standard form of IGC and is called infinite dilution IGC (ID-IGC). ${ }^{27}$

However, because the SEA can accurately control the coverage of probe molecules on the surface, it can measure so-called surface energy profiles, i.e. the surface energy as a function of probe coverage. This is termed finite dilution IGC (FD-IGC), ${ }^{28}$ and allows the characterisation of not just high energy sites but also sites with lower probe-surface binding energy. Such sites tend to predominate only as the probe coverage is increased. Thus, a typical surface energy profile shows a high surface energy value at low coverage which is representative of high energy sites. However, at the coverage increases, the surface energy falls gradually before plateauing at a value which related to the low energy sites.

One factor which is not generally discussed is that probe-probe interactions may become important at high probe coverage. This manifests itself in some surface energy profiles (see SI figure S6) where the surface energy plateaus before beginning to increase with coverage. This is generally accompanied by peak fronting in the chromatograms which has been associated with probe-probe interactions by Ho et al. ${ }^{29}$ Where such increases were observed, we removed the relevant data points and only analysed data where the measured surface energy falls or stays constant with increasing probe coverage. While this should minimise the effect of probe-probe 
interactions, we cannot avoid the fact that surface energy values in the high coverage regime may be slightly over estimated.

In practise, the retention volume is measured for a set of alkane probes and the surface energy calculated using equation 5 . This is repeated at a range of coverages to produce a surface energy profile. In order to accurately relate injection volumes to surface coverage, the specific surface area $\left(S_{B E T}\right)$ of a sample must be known. A description of the specific surface area measurement is given in the supplemental information. We note that the Dorris-Gray method was initially developed for infinite dilution systems. ${ }^{24}$ However, in recent years it has become standard practise to extend the Dorris-Gray method to finite dilution systems like the ones in this work. ${ }^{11,15,30,31}$

\section{RESULTS AND DISCUSSION}

\section{Basic characterisation}

In order to obtain a broad picture of the surface energy of graphite, we purchased graphite powder from nine suppliers with the aim of comparing their surface energetics (information on the graphite types is given in the SI). First, we carried out some very basic characterisation of the various graphite types. To characterise the size of the graphite flakes used in this study, SEM was conducted on each sample to measure the mean flake dimensions. Figure 1A shows a representative SEM image of one of the graphite samples (9-Henglide). This image appears to show the graphite flakes to be particulate in nature. However, more detailed SEM examination shows that the flakes are not monolithic but rather are clearly made of stacked platelets as shown in figure 1B These platelets were observed in all samples and were $<1 \mu \mathrm{m}$ thick in all cases.

From statistical analysis of the SEM images, we measured the mean length of the graphite flakes, finding values in the range 10-1000 $\mu \mathrm{m}$ as shown in figure $1 \mathrm{C}$ (we define $L$ as the flakes maximum dimension, see SI). We also measured the specific surface area of the graphite samples (see SI). These values were clustered between 5 and $15 \mathrm{~m}^{2} / \mathrm{g}$, a much smaller range than observed for the flakes lengths (figure 1C). The specific surface area of a thin platelet of area, $A$, perimeter length, $P$, and thickness, $t$, is given by

$$
S_{B E T}=\frac{2 A+P t}{\rho A t}=\frac{2}{\rho t}+\frac{P}{\rho A}
$$


where $\rho$ is the platelet density. Here the first term represents the basal plane contribution while the second term represents the edge area. In the SI, we model the platelets as rhombuses with longest dimension, $L$, and size $L / k$ in the perpendicular direction where $k$ is the aspect ratio. This allows us to approximate the perimeter to area ratio as $P / A=4 \sqrt{1+k^{2}} / L$, giving $S_{B E T}=\frac{2}{\rho t}+\frac{4 \sqrt{1+k^{2}}}{\rho L}$

Because the data in figure 1C suggests $S_{\mathrm{BET}}$ to be roughly independent of $L$, this implies the edge contribution to be relatively small and the platelet thickness to be also approximately independent of $L$. From the mean value of $\left\langle S_{B E T}\right\rangle=8.2 \mathrm{~m}^{2} / \mathrm{g}$, we can estimate the mean platelet thickness to be close to $100 \mathrm{~nm}$ for all graphite types.

To investigate the quality of the different graphite types, we conducted Raman spectroscopy. Typical spectra for two of the samples are shown in figure 1D with the rest shown in the SI. Raman spectroscopy is a well-established technique for the characterisation of defects in $\mathrm{sp}^{2}$ carbon based materials such as graphite, graphene and carbon nanotubes. ${ }^{32-34} \mathrm{We}$ can use the ratio of the D-peak to G-peak intensity $\left(I_{D} / I_{G}\right)$ as a measure of the defect content in a sample, ${ }^{32}$ since the D-band is only observed in samples containing defects. ${ }^{35}$ In Raman spectroscopy, a defect is defined as any breaking of the symmetry of the graphene lattice, such as vacancy sites ${ }^{36,37}$ and edges. ${ }^{38,39}$ A more detailed discussion on the origin of Raman peaks is given by Beams et al. ${ }^{40}$ Among the graphite samples studied, the $I_{D} / I_{G}$ ratio varies from less than 0.01 for Northern to 0.26 for Henglide graphite. As shown in the SI, $I_{D} / I_{G}$ scales linearly with $1 / L$ with a positive intercept, behaviour which implies the basal plane defect concentration to be roughly constant over these samples.

Finite-dilution inverse gas chromatography measurements

In order to ascertain how the surface energy of different commercial graphites differ from each other, the dispersive surface energy $\left(\gamma_{d}\right)$ profiles were measured using coverage-dependent IGC. Such $\gamma_{d}$ profiles are measured by injecting a series of n-alkane probes from hexane to nonane at selected surface coverages. Surface coverage $(\phi)$ is calculated as a fraction of the monolayer capacity of the sample from specific surface area measurements (see SI for results). Shown in figure 2A are two alkane plots, portraying the Gibbs free energy of adsorption $\left(\Delta G_{\text {probe }}\right)$ as a function of alkane carbon number, $n$, measured at low and high 
probe coverage (graphite sample Timcal). At any coverage $\gamma_{d}$ is calculated using the slope of the alkane plot and the Doris-Gray formula as shown in equation 5. This process is repeated for all coverages to complete the $\gamma_{d}$ profile. Figure 2B shows the $\gamma_{d}$ profiles of two of the graphite samples used in this study. The profiles have the usual concave shape, with surface energy falling with increasing probe coverage ${ }^{41}$ from the low- $\phi$ value of $\gamma_{d, \phi=0}$, before reaching a coverage-independent plateau at large coverage. ${ }^{42-45}$ This plateau value is defined as the full coverage surface energy, $\gamma_{d, \phi=1} \cdot{ }^{46}$ Significant variations in surface energy profiles were observed from sample to sample.

For the graphites studied here, we observed values of $\gamma_{d, \phi=0}$ in the range $125-175 \mathrm{~mJ} / \mathrm{m}^{2}$ while $\gamma_{d, \phi=1}$ values were in the range $50-75 \mathrm{~mJ} / \mathrm{m}^{2}$ (figure $2 \mathrm{C}$ ). The measured values of $\gamma_{d, \phi=0}$ compare well to previous infinite dilution IGC measurements of graphite. For example, Balard et al found the ID-IGC $\gamma_{d}$ for as-received commercial graphite to be $115 \mathrm{~mJ} / \mathrm{m}^{2}{ }^{47}$ while Papirer et al measured two commercial graphite samples and found $\gamma_{d}$ to be $105 \mathrm{~mJ} / \mathrm{m}^{2}$ and $279 \mathrm{~mJ} / \mathrm{m}^{2}{ }^{48}$ In addition, Donnet et $\mathrm{al}^{49}$ measured a $\gamma_{d}$ value of $134 \mathrm{~mJ} / \mathrm{m}^{2}$ and Park et al ${ }^{13}$ measured two graphite samples to be $119 \mathrm{~mJ} / \mathrm{m}^{2}$ and $120 \mathrm{~mJ} / \mathrm{m}^{2}$ (adjusted for temperature). However, the full coverage values $\left(\gamma_{d, \phi=1}\right)$ are far lower than the literature ID-IGC values quoted above and are more comparable to values of surface energy measured by contact angle ${ }^{50}$ or suggested by solubility measurements ${ }^{51}$ which tend to fall in the range $50-125 \mathrm{~mJ} / \mathrm{m}^{2} .^{52-54}$ This is consistent with the idea that these techniques give an average over the whole surface and not just the highest energy sites.

We note that it is challenging to compare literature values to one another in great detail due to the different experimental conditions used in each set of experiments. Such differences make it hard to differentiate the effects of different experimental conditions from those due to the inhomogeneous nature of graphite's surface. ${ }^{4}$ We address these problems by insuring that all samples have the same total surface area $\left(0.5 \mathrm{~m}^{2}\right)$ in the column. In addition, the same coverage values and combination of alkane probes used for all measurements. To the best of our knowledge this is the first paper to show a comprehensive comparison of the $\gamma_{d}$ profiles of commercial graphites. 
In the literature, $\gamma_{d}$ profiles for energetically heterogeneous surfaces are often empirically fitted to an exponential decay function: ${ }^{30,55}$

$\gamma_{d}(\phi)=\gamma_{d, \phi=1}+\left(\gamma_{d, \phi=0}-\gamma_{d, \phi=1}\right) e^{-\phi \mid \phi_{0}}$

where $\gamma_{d, \phi=1}$ is the dispersive surface energy at full coverage, $\gamma_{d, \phi=0}$ is the dispersive surface energy at zero coverage, and $\phi_{0}$ is a constant which describes how fast the surface energy decays with increasing coverage. However, we have found that a stretched/compressed exponential tends to match the data much better and give more consistent results (we will justify this in more detail below and in the SI):

$\gamma_{d}(\phi)=\gamma_{d, \phi=1}+\left(\gamma_{d, \phi=0}-\gamma_{d, \phi=1}\right) e^{-\left(\phi / \phi_{0}\right)^{k}}$

where $k$ is the stretching (when $0<k<1$ ) or compression $(k>1)$ exponent. We have fitted all coverage-dependent surface energy data to equation $7 \mathrm{~b}$, extracting $\gamma_{d, \phi=1}, \gamma_{d, \phi=0}, \phi_{0}$ and $k$. As shown in figure 2B good fits were obtained with values of $k$ between 0.85 and 1.8 (see SI for all fits).

Shown in figure $2 \mathrm{C}$ is a plot of $\gamma_{d, \phi=1}$ versus $\gamma_{d, \phi=0}$, showing no simple correlation between these parameters suggesting that they may be independent of each other. This implies that high energy sites (probed at low coverage) on the sample surface are not representative of the surface as a whole (probed at high coverage) and so carry little information about the intrinsic surface properties. This shows the importance of coverage-dependent IGC compared to ID-IGC. We have plotted both $\gamma_{d, \phi=1}$ and $\gamma_{d, \phi=0}$ (both extracted from the fits to equation $7 \mathrm{~b}$ ) versus $\phi_{0}$ in figure 2D. Here, $\gamma_{d, \phi=1}$ appears to be independent of $\phi_{0}$. However, we see a clear correlation between $\gamma_{d, \phi=0}$ and $\phi_{0}$ as shown in figure 2D. This implies that these two parameters are related and have their origins in the same phenomena.

\section{Simulation of finite-dilution inverse gas chromatography response}

In order to interpret the finite-dilution inverse gas chromatography data shown in figure $2 \mathrm{~B}-\mathrm{D}$, it is important to better understand the nature of the sites where the probe molecules bind to the graphitic surface. Such understanding will in turn shed light on the details of the heterogeneity of the surface chemistry of graphite. Recently, a method has been reported which allows the surface energy coverage profile to be simulated based on inputted energetic 
distributions of binding sites (see methods). Here we use such simulations to gain some broad insights into the relationships between $\gamma_{d, \phi=1}, \gamma_{d, \phi=0}, \phi_{0}$ and the energetic distribution of binding sites.

We begin by considering one population of binding sites whose contribution to the surface energy can be described by a normalised Gaussian distribution:

$$
N\left(\gamma_{d}\right)=\frac{1}{\Delta \gamma \sqrt{2 \pi}} e^{-\left(\gamma_{d}-\gamma_{0}\right)^{2} /(2 \Delta \gamma)^{2}}
$$

which is centred at $\gamma_{0}$ with width $\Delta \gamma$. Three example distributions are shown in figure $3 \mathrm{~A}$. We used the simulation techniques initially described by Jefferson et $\mathrm{al}^{56}$ and Smith et $\mathrm{al}^{57}$ to calculate the surface energy versus coverage profiles for a range of such distributions with various combinations of $\gamma_{0}$ and $\Delta \gamma$, with examples in figure $3 \mathrm{~B}$ corresponding to the distributions in figure 3A. In all cases, the simulated profiles took the same general form as experimental data with the surface energy decreasing with coverage. However, all simulated curves were convex in shape with none showing the exponential-like shape which tends to be found experimentally. Nevertheless, it is still possible to gain some insights from these single exponential coverage profiles. For example, we found the full coverage surface energy, $\gamma_{d, \phi=1}$ , to be exactly equal to the centre of the distribution, irrespective of the distribution width (figure 3C). Perhaps more interestingly, we found the difference between the zero- and fullcoverage surface energies to scale with distribution width approximately as

$$
\gamma_{d, \phi=0}-\gamma_{d, \phi=1} \approx 0.045 \times \Delta \gamma^{2}
$$

where the surface energies are in $\mathrm{mJ} / \mathrm{m}^{2}$. Although this surface energy difference depends very weakly on the distribution centre, we find the approximation holds well for all values of $\gamma_{0}$ between 60 and $200 \mathrm{~mJ} / \mathrm{m}^{2}$ with deviations of no more than $25 \%$. This relationship reflects the fact that the deepest sites fill up first, allowing us to make two simple observations: The value of $\gamma_{d, \phi=0}$ reflects the contribution to the surface energy of those sites which bind the probe molecules most strongly (i.e. the deepest sites) while $\gamma_{d, \phi=1}$ is a measure of the mean contribution to the surface energy.

Because of the lack of agreement between the shapes of simulated and experimental coverage profiles, we hypothesised that real experimental data is rarely, if ever, associated with a single 
population of binding sites. We thus carried out a range of simulations with two normalised Gaussians, representing populations $N_{L E}\left(\gamma_{d}\right)$ and $N_{H E}\left(\gamma_{d}\right)$ such that the total normalised distribution of binding sites is given by $N_{T}\left(\gamma_{d}\right)=\left(1-f_{H E}\right) N_{L E}\left(\gamma_{d}\right)+f_{H E} N_{H E}\left(\gamma_{d}\right)$, where $f_{H E}$ is the fraction of HE sites. Here the subscripts represent "High Energy" and "Low Energy" such that $\gamma_{0, L E}<\gamma_{0, H E}$. An example of a pair of such Gaussians and their sum is given in figure 3E.

We first investigated whether such a two-Gaussian distribution can give an exponential-like concave surface energy versus coverage profile by calculating a number of profiles for pairs of distributions with different combinations of $\gamma_{0}$ and $\Delta \gamma$. We found exponential-like coverage plots so long as the high energy distribution was relatively broad (roughly $\Delta \gamma>0.3 \gamma_{0}$ ), a condition that is fulfilled by the example distributions in figure $3 \mathrm{E}$.

We then systematically calculated surface energy versus coverage profiles for pairs of distributions, keeping the positions and widths constant for each pair but varying the fraction of high energy sites $\left(f_{H E}\right)$. A typical sub-set of such profiles is given in figure $3 \mathrm{~F}$ (calculated from the distributions in figure $3 \mathrm{E}$ ). In general, such profiles are exponential-like (obviously except when $f_{H E}=0$ or 1 ). However, we note that such simulated profiles cannot be well-fitted to exponential decays but are much better described by stretched exponentials $(k<0)$ as illustrated in figure $3 \mathrm{~F}$ inset and SI. From the profiles, we have extracted $\gamma_{d, \phi=1}, \gamma_{d, \phi=0}$ and $\phi_{0}$ (as well as $k$ ). Shown in figure $3 \mathrm{G}$ is a graph of $\gamma_{d, \phi=1}$ and $\gamma_{d, \phi=0}$, extracted from the profiles in figure $3 \mathrm{~F}$, plotted versus the fraction of high energy sites, $f_{H E}$. This data shows that $\gamma_{d, \phi=1}$ is just the weighted mean of the centres of the two Gaussian distributions such that: $\gamma_{d, \phi=1}=\left(1-f_{H E}\right) \gamma_{0, L E}+f_{H E} \gamma_{0, H E}$. However, $\gamma_{d, \phi=0}$ is slightly less straightforward to analyse, rising sharply as $f_{H E}$ is increased, and approaching the value of $\gamma_{d, \phi=0}$ appropriate to the $\mathrm{HE}$ sites once the fraction of HE sites passes $\sim 0.4$ in this case. We note that this result is consistent with the single Gaussian distribution case because it shows that $\gamma_{d, \phi=0}$ is largely controlled by the energy of the deepest sites. This of course means that $\gamma_{d, \phi=0}$ should be particularly sensitive to the width of the HE Gaussian distribution.

We have also plotted $\phi_{0}$ (extracted from fits to equation 7b) versus the fraction of high energy sites, $f_{H E}$, in figure $3 \mathrm{H}$. This graph is particularly interesting as it shows a linear relationship, $\phi_{0}=\beta f_{H E}$, for $f_{H E}<0.4$ in this case. Fitting the linear portion gives $\beta=1.1$ in this situation. This 
is potentially a very important relationship as it shows that, in principle, the population of high energy sites can be extracted directly from the stretched/compressed exponential fit of the data. However, there is a significant discrepancy between the simulated two-Guassian data and the real experimental data reported in figure 2. The data in figure 2D shows $\gamma_{d, \phi=0}$ to decrease with $\phi_{0}$ (i.e. with $f_{H E}$ ) while the simulations (figure $3 \mathrm{H}$ inset) show the opposite trend. This trend in the simulation data arises because the higher energy distribution is broader than the low energy distribution, a necessary condition of finding concave, exponential-like coverage curves (see above and SI). This strongly suggests that our experimental data cannot be described by two Gaussian distributions but actually require a third distribution. This can be understood by considering the combination of a low energy distribution ( $L E)$ and two high energy distributions, $H E, 1$ and $H E, 2$, such that $H E, 1$ represents a small fixed population of deep sites while $H E, 2$ represents somewhat shallower sites (although not as shallow as the $L E$ sites). When the population of $H E, 2$ is small, the deep sites associated with $H E, 1$ will dominate, resulting in a high value of $\gamma_{d, \phi=0}$. However, as the population of $H E, 2$ is increased, these sites will come to dominate, thus reducing the overall value of $\gamma_{d, \phi=0}$, as observed in figure $2 \mathrm{D}$.

We can see this quantitatively by simulating the surface energy versus coverage plots for three Gaussian distributions, labelled $L E, H E, 1$ and $H E$, 2, with a range of relative populations (figure 3I-L). For reasons which will become clear below, we fix the population of $H E, 1$ at an arbitrary value of $5 \%$ of $L E$, but allow the population of $H E, 2$ to vary. Because figure $2 \mathrm{D}$ shows $\gamma_{d, \phi=1}$ to be roughly constant over all graphites, and because figure $2 \mathrm{G}$ implies $\gamma_{d, \phi=1}$ to be the weighted mean of the distribution centres, we set the means of the $L E$ and $H E, 2$ distributions as $60 \mathrm{~mJ} / \mathrm{m}^{2}$ (because the population of $H E, 1$ is small, its position will not significantly affect the overall value of $\left.\gamma_{d, \phi=1}\right)$. We then used trial and error to find values of the remaining parameters $\left(\gamma_{0, H E 1}, \Delta \gamma_{L E}, \Delta \gamma_{H E 1}, \Delta \gamma_{H E 2}\right)$ such that, when the $H E, 2$ population (i.e. $\left.f_{H E 2}\right)$ is varied, we could reproduce the experimental data shown in figure $2 \mathrm{D}$.

We found this to be the case using the distributions shown in figure 3I. The low energy ( $L E)$ distribution had position and width of 60 and $5 \mathrm{~mJ} / \mathrm{m}^{2}$ while $H E, 1$ and $H E, 2$ had positions of 92 and $60 \mathrm{~mJ} / \mathrm{m}^{2}$ respectively with both widths of $20 \mathrm{~mJ} / \mathrm{m}^{2}$. Also shown in figure 3I (black curve) is an example of an overall distribution with fractions of each component: $f_{L E}=0.9$, $f_{H E I}=0.045$ and $f_{H E 2}=0.055$. We calculated the surface energy versus coverage curves for 
combinations of these distributions, keeping the $H E, 1$ to $L E$ population ratio at $5 \%$ but varying the $L E$, 2 population $\left(f_{H E 2}\right)$. Two example curves are given in figure $3 \mathrm{~J}$. As shown in the inset, such curves can be much better fit to stretched exponentials (equation $7 \mathrm{~b}$ ) than exponential curves (equation 7a). From the stretched exponential fits, we extracted $\gamma_{d, \phi=0}$ and $\gamma_{d, \phi=1}$, which we plotted versus the total fraction of high energy sites $\left(f_{H E}=f_{H E 1}+f_{H E 2}\right)$ in figure $3 \mathrm{~K}$. This graph shows $\gamma_{d, \phi=0}$ to fall with fraction of high energy sites, fundamentally different behaviour to that observed for the two-Gaussian distributions in figure $3 \mathrm{G}$, but consistent with the data in figure $2 \mathrm{D}$. We note that, although the data is plotted versus $f_{H E 1}+f_{H E 2}$, this decay in $\gamma_{d, \phi=0}$ is directly related to $f_{H E 2}$. As shown in figure $3 \mathrm{~K}$ (inset) $\gamma_{d, \phi=0}$ falls empirically with $f_{H E 2}$ as a single-exponential decay, a fact which will be important later.

From the fits, we also extracted the decay constant, $\phi_{0}$, which we plot against the total fraction of high energy sites $\left(f_{H E}=f_{H E 1}+f_{H E 2}\right)$ in figure $3 \mathrm{~L}$. As with the two-Gaussian simulations described in figure $3 \mathrm{H}$, we find linearity for small values of $f_{H E}$, described by $\phi_{0}=\beta f_{H E}$, although here $\beta=0.35$, significantly different to the value of $\beta=1.1$ found in figure $3 \mathrm{H}$ for the 2-Gaussian simulations. We believe the actual value of $\beta$ is determined by the details of the overall binding energy distribution, essentially making it an intrinsic material parameter. This result is important as it shows that, even for a three-Guassian system, the decay constant is a measure of the fraction of high energy sites. Shown in figure $3 \mathrm{~L}$ inset is a graph of $\gamma_{d, \phi=0}$ and $\gamma_{d, \phi=1}$, plotted versus $\phi_{0}$, which matches very well to the experimental data in figure 2D. This clearly shows that the overall surface energy data for the set of nine graphite's studied experimentally is consistent with the probe molecules binding to three distinct types of binding site which can be simulated by the three distributions shown in figure 3I.

\section{Modelling surface energy data}

With this in mind, we can consider the physical nature of the three types of binding sites discussed above. We believe the high-energy sites probed at low-coverage are associated with defects in the graphitic lattice. These can either be associated with the flakes edges or be basal plane defects such as point defects. Conversely, the dominant low-energy sites probed at highcoverage represent the basal plane in the absence of defects. We would expect the population of basal plane defect binding sites to be low relative to the number of graphitic basal plane binding sites. In the ideal case where all of the studied graphite types were identical and varied 
only in flake size, we would expect the ratio of these populations to be fixed. Conversely, we would expect the number of edge binding sites to scale with flake size and so vary among the samples studied. Thus, we can associate the $L E, H E, 1$ and $H E, 2$ distributions with basal plane, basal plane defects and edge binding sites respectively.

If this model is correct, in the ideal case we would associate $\gamma_{d, \phi=1}$ with the intrinsic surface energy of graphite in the absence of defects or edges. However, this may not be exactly the case here as the graphite used in this study was not exceptionally clean. In addition, the annealing process applied to the graphite was not particularly aggressive and was unlikely to result in a pristine graphitic surface. This means the values of $\gamma_{d, \phi=1}$ obtained may not correlate exactly with the true surface energy of graphite but more accurately reflect the surface energy of a graphitic surface contaminated with hydrocarbons. ${ }^{54}$ In any case, within this model, we would expect $\gamma_{d, \phi=1}$ to be independent of the flake dimensions. This is shown in figure 4A where we plot $\gamma_{d, \phi=1}$ versus mean flake length for nine samples of commercial graphite. We find the data to be size-independent with all data clustered around a mean of $\left\langle\gamma_{d, \phi=1}\right\rangle$ $=63 \pm 7 \mathrm{~mJ} / \mathrm{m}^{2}$.

We now turn to $\gamma_{d, \phi=0}$ and $\phi_{0}$. Within our model, we would expect the low coverage behaviour, and so $\gamma_{d, \phi=0}$ and $\phi_{0}$, to be controlled by the defect population. It is most easy to understand $\phi_{0}$. This parameter represents the coverage where the high energy sites (defects) become saturated with probe molecules and as shown above, should scale with the number of defects in the system $\left(\phi_{0}=\beta f_{H E}\right)$. We can test the relationship between $\phi_{0}$ and defect content by plotting the former parameter as a function of $I_{D} / I_{G}$. As described above, $I_{D} / I_{G}$ is measured by Raman spectroscopy and is a measure of the graphite defect content. As shown in figure 4B, the linear trend indicates that $\phi_{0}$ is indeed related to the amount of defects present.

If $\phi_{0}$ scales with defect content, then we would expect smaller flakes to have larger values of $\phi_{0}$, simply because smaller flakes have more edge defects. Plotted in figure 4C is a graph of $\phi_{0}$ versus mean flake length, L, for nine commercial graphite types. This graph clearly shows the expected behaviour with a well-defined fall-off of $\phi_{0}$ with increasing L. 
We can quantitatively model $\phi_{0}$ as the fraction of graphitic surface area occupied by probe molecules when all defects have been saturated with probe molecules. We must consider a combination of both edge and basal plane defects. We assume that there are $N_{D, B} / A$ defects per unit area of basal plane and that around each defect is an area $A_{H E}$ which acts as a high energy region for the adsorption of probe molecules. We note that this area might be significantly larger than the actual defect size and represents the area where the carbon atoms have deviated from their defect-free positions. In addition, the whole of the edge region of a flake also behaves as an array of high energy sites for probe molecules. Then, by treating the flake as a platelet of area, $A$, perimeter length, $P$, and thickness, $t$, we can write:

$$
\phi_{0}=\beta f_{H E}=\beta \frac{\left(N_{D, B} / A\right) A_{H E} 2 A+P t}{2 A+P t}
$$

We note that $A_{H E}\left(N_{D, B} / A\right)$ represents the fraction of basal plane area where the surface energy deviates from that of graphitic carbon due to the presence of defects. As described above (and in the SI), modelling the platelets as rhombuses gives a relationship between $P$ and $A$. Then, in the limit where the platelet is thin and so the edge area can be neglected (see above), this simplifies to

$$
\phi_{0} \approx \beta \frac{N_{D, B}}{A} A_{H E}+2 \beta \sqrt{1+k^{2}} \frac{t}{L}
$$

The inset in figure $4 \mathrm{C}$ shows a linear relationship between $\phi_{0}$ and $1 / L$ as predicted by equation 10b. Interestingly, the lack of scatter suggests all nine graphite types to have similar values of $t, k$ and $A_{H E}\left(N_{D, B} / A\right)$. The presence of a constant flake thickness is consistent with the specific surface area measurements, while the constancy of $A_{H E}\left(N_{D, B} / A\right)$ is consistent with the length dependence of the Raman data (see SI). In addition, $k$ is known to be constant from SEM measurements which show a mean value of $k=1.6$ over all samples. That the data is consistent with values of $\beta$ and $A_{H E}\left(N_{D, B} / A\right)$ which are roughly constant over all the graphite samples implies that the nature of both edges and basal plane defects is the same for the range of graphite types studied. While it would be possible to use the mean value of $t$ extracted from BET analysis to find $\beta$ and $A_{H E}\left(N_{D, B} / A\right)$ from the linear fit, we demonstrate a better method below.

It is possible to perform a more quantitative analysis by combining equations $6 \mathrm{a}$ and 10a to eliminate $t$ and then using the relationship $P / A=4 \sqrt{1+k^{2}} / L$ to give: 
$\left[\frac{\rho S_{B E T} \phi_{0} L}{4 \sqrt{1+k^{2}}}\right]=\beta \times\left(N_{D, B} / A\right) A_{H E} \times\left[\frac{\rho S_{B E T} L}{4 \sqrt{1+k^{2}}}-1\right]+\beta$

Because all the parameters in the square-bracketed terms are known or have been measured in this work, it is possible to parameterise the data in accordance with equation 11 and plot a graph which should give linear behaviour. This is shown in figure 5 and does indeed show the expected behaviour with the line representing linearity. From this fit, we can extract a value of $\beta=9 \pm 2$ and $A_{H E}\left(N_{D, B} / A\right)=(0.4 \pm 0.1) \%$. As described above, we believe the value of $\beta$ to be an intrinsic material property which depends on the overall surface energy distribution including contributions from basal plane, basal plane defects and edges. We can use this value of $A_{H E}\left(N_{D, B} / A\right)$ to estimate the areal basal plane defect density, $N_{D, B} / A$. We assume, that the presence of a point defect effects the surface energy locally over a distance of $\sim 1 \mathrm{~nm}$, giving $A_{H E} \sim 3 \mathrm{~nm}^{2}$. This allows us to estimate $N_{D, B} / A \sim 10^{15}$ defects $/ \mathrm{m}^{2}$, close to the population of neutral point defects measured for graphene by Barreiro et al $\left(2.5 \times 10^{15}\right.$ defects $\left./ \mathrm{m}^{2}\right) .{ }^{58}$

Shown in figure $4 \mathrm{D}$ is a plot of $\gamma_{d, \phi=0}$ versus $L$ which shows a well-defined monotonic increase. This is consistent with the data in figures $2 \mathrm{D}$ and $3 \mathrm{~K}$ and can be understood as follows. When $L$ is very large, flake edges are relatively uncommon and the fraction of edge defects $\left(f_{H E, 2}\right)$ becomes very low. This means that $\gamma_{d, \phi=0}$ is dominated by the relatively deep sites associated with the basal plane defect $(H E, 1)$ distribution. However, as $L$ falls, the population of edge defects $\left(f_{H E 2}\right)$ increases until for very small flakes, the basal plane defect content becomes negligible and edge defects predominate. Then, the $H E, 2$ (edge) distribution, with its shallower sites controls the low coverage surface energy, resulting in a reduction of $\gamma_{d, \phi=0}$. As a result, $\gamma_{d, \phi=0}$ increases steadily from its low- $L$, edge defect dominated value of $\sim 130 \mathrm{~mJ} / \mathrm{m}^{2}$ to its high$L$ basal plane defect dominated value of $\sim 180 \mathrm{~mJ} / \mathrm{m}^{2}$.

Knowledge of the numerical value of $A_{H E}\left(N_{D, B} / A\right)$ allows us to test our understanding of the data in figure 4D more deeply. We have proposed that $\gamma_{d, \phi=0}$ is controlled by edge sites at low $L$ and by basal plane defects at high $L$. If this is the case, we would expect the populations of edge and basal plane defects to be equal at an intermediate value of $L$. We can calculate this value by equating the basal plane area affected by defects with the edge area: $A_{H E}\left(N_{D, B} / A\right) \times 2 A=P t$. Then, using the expression for $P / A$ given above and taking $t \sim 100 \mathrm{~nm}$, $k=1.6$ and $A_{H E}\left(N_{D, B} / A\right)=0.4 \%$, we can work out the flake length where basal plane (BD) and 
edge defects (ED) populations are equal. This works out as $L_{B D=E D}=2 t \sqrt{1+k^{2}} /\left[A_{H E}\left(N_{D, B} / A\right)\right] \approx 100 \mu \mathrm{m}$, a value which lies in the middle of the range where $\gamma_{d, \phi=0}$ is transitioning from a low to a high value. This is exactly as expected given our explanation as described above.

We can extend this analysis of the $\gamma_{d, \phi=0}$ by noting that, as shown in figure $3 \mathrm{~K}$ (inset), simulated values of $\gamma_{d, \phi=0}$ fall empirically with $f_{H E 2}$ as a single-exponential decay. Because the fractional population of edge defects scales approximately as $f_{H E 2} \approx P t / 2 A \propto 1 / L$ (see equation 10), this implies that $\gamma_{d, \phi=0}$ should scale with $L$ as $\gamma_{d, \phi=0}=\gamma_{d, \phi=0}(L=0)+\left[\gamma_{d, \phi=0}(L=\infty)-\gamma_{d, \phi=0}(L=0)\right] e^{-L_{B D=E D} \ln 2 / L}$. We can justify the form of the exponential by noting that, when written this way, $L_{B D=E D}$ represents the flake length where $\gamma_{d, \phi=0}$ is midway between its small- $L$ and large- $L$ limits. This is consistent with the definition that $L_{B D=E D}$ is equal to flake length where the populations of edge and basal plane defects are equal. As shown by the solid line in figure 4D, the data is indeed consistent with this functional form with fitting parameters $\gamma_{d, \phi=0}(L=0)=125 \mathrm{~mJ} / \mathrm{m}^{2}, \gamma_{d, \phi=0}(L=\infty)=180 \mathrm{~mJ} / \mathrm{m}^{2}$ and $L_{B D=E D}$ $=86 \mu \mathrm{m}$. The first two fit parameters represent the intrinsic edge and basal plane defect surface energies. In addition, the fit value of $L_{B D=E D}$ is very close to the value calculated above. This analysis supports the association between the $H E, 2$ defects and the nanosheet edges and highlights the agreement between simulation and experimental results.

This allows us to consider the surface energy profiles expected for graphene nanosheets. Because we expect the intrinsic basal plane surface energy to be similar to that of graphite, we expect $\gamma_{d, \phi=1} \approx 60 \mathrm{~mJ} / \mathrm{m}^{2}$. In addition, assuming $A_{H E}\left(N_{D, B} / A\right)$ is similar for graphene and graphite and taking $t \sim 2 \mathrm{~nm}$ and $k \sim 1.6$, typical values for liquid exfoliated graphene nanosheets, ${ }^{9}$ we can work out $L_{B D=E D} \approx 2 \mu \mathrm{m}$ for graphene. As most liquid exfoliated graphene nanosheets are smaller than this, with lengths in the range $\mathrm{e}^{8,9} 0.1-1 \mu \mathrm{m}$, we expect $\gamma_{d, \phi=0}$ to be controlled by edge defects. This allows us to predict that graphene nanosheets will display values of $\gamma_{d, \phi=0}$ approaching $125 \mathrm{~mJ} / \mathrm{m}^{2}$. In addition, because the edge defect population will be relatively high, we expect $\phi_{0}$ values to be higher for graphene samples than for graphite. 


\section{Comparison with literature}

This works suggests $\gamma_{d, \phi=1}$ to represent the intrinsic dispersive component of the surface energy of graphite. To compare the $\gamma_{d, \phi=1}$ measurements from this study to literature values, we extracted literature data for graphite surface energies from contact angle measurements, ${ }^{2,50,52,54,59-61}$ liquid exfoliation, ${ }^{53}$ frictional sliding with adsorption measurements, ${ }^{62}$ crystal cleavage,${ }^{63,64}$ Krypton adsorption ${ }^{65}$ and theoretical calculations. ${ }^{3,66-68}$ These are shown in ascending order of surface energy as shown in figure 6. Here we label the sample number as $n$, where $n$ ascends from 1 to 34 . It should be noted that the data in figure 6 doesn't necessarily differentiate between dispersive surface energy $\left(\gamma_{d}\right)$ and total surface energy $\left(\gamma_{T}\right)$ but the difference between these will be low for materials like graphite where specific interactions are minimal in comparison to $\gamma_{d}$. The energy values often used in crystal cleavage experiments quote the energy to cleave one crystal from another. If this was the case we divided the energy value by 2 to get the surface energy (from the definition of surface energy). This literature data extends over a very wide range, from 30 to $1000 \mathrm{~mJ} / \mathrm{m}^{2}$, with the data found here clustered at the low end.

Assuming this literature data represents a random subset of the true distribution of surface energy values, then it can be shown (see SI) that the data, as plotted in figure 6 represents the integral of the overall distribution of measured graphite surface energies, $n\left(\gamma_{\text {graphite }}\right) \propto \int g d \gamma_{\text {graphite }}$, where $g$ represents the overall distribution. The kink in the $n v s$. $\gamma_{\text {graphite }}$ curve at $\gamma_{\text {graphite }} \sim 85 \mathrm{~mJ} / \mathrm{m}^{2}$ suggests the global distribution of graphite surface energies to include two components, one in the range $30-100 \mathrm{~mJ} / \mathrm{m}^{2}$ and the other in the range $70-1000$ $\mathrm{mJ} / \mathrm{m}^{2}$. We found that we could fit the $n\left(\gamma_{\text {graphite }}\right)$ data to the integral of the sum of two lognormal distributions, $g_{L N 1}$ and $g_{L N 2}$. Because the integral of a log-normal function is an error function, this is equivalent to fitting to the sum of two error functions, $\operatorname{erf}_{1}$ and $\operatorname{erf}_{2}$ as shown by equation 12 :

$$
n\left(\gamma_{\text {graphite }}\right) \propto \int\left(g_{L N 1}+g_{L N 1}\right) d \gamma_{\text {graphite }} \propto A \times \operatorname{erf}_{1}\left(\gamma_{\text {graphite }}\right)+B \times \operatorname{erf}_{2}\left(\gamma_{\text {graphite }}\right)
$$

where A and B are weighting factors. As can be seen from figure 6, the fit is very good. From the fit parameters, we can reconstruct $g_{L N 1}$ and $g_{L N 2}$ and so the overall global distribution $g=g_{L N 1}+g_{L N 2}$. Shown in figure 6 inset are $g, g_{L N 1}$ and $g_{L N 2}$. It is interesting that there is a 
clear division of data types over the two distributions. Over the energy range associated with $g_{L N 1}$, we have data from contact angle measurements, data extracted from liquid phase exfoliation experiments and the IGC data taken from $\gamma_{d, \phi=1}$. These are all liquid based techniques associated with full coverage. Conversely, over the energy range associated with $g_{L N 2}$, we have data from crystal cleavage, sliding as well as theory results. These techniques have in common the absence of liquid. It is not clear why this should be. However, it is clear that the spread of published surface energy data may have more structure to it than was previously thought.

\section{CONCLUSION}

In this work we have used finite dilution inverse gas chromatography to measure the surface energy of a range of graphites as a function of the degree of coverage of the probe molecule. As the coverage is increased the measured surface energy always falls before plateauing at high coverage. We find the high-coverage plateau to be invariant with graphite type with a value of $63 \pm 7 \mathrm{~mJ} / \mathrm{m}^{2}$, which we associate with the basal plane surface energy. The low coverage surface energy increases monotonically with graphite flake size, allowing us to associate it with defects which are located both at flake edges and as point defects in the basal plane. Using a combination of simulations and simple analytical models, we could fit the data, allowing is to separate out the contributions of edge and point defects.

While this paper has discussed the surface energy of graphite, the methods discussed here are applicable to the analysis of FD-IGC data for any material. Most obviously, the ideas expressed here could be directly transferred to the measurement of the surface energy of other layered crystals such as $\mathrm{BN}$ or $\mathrm{MoS}_{2}$. In addition, these methods will be useful for analysing the surface energy of exfoliated 2D materials such as graphene.

\section{METHODS}

\section{Experimental}

Graphite samples were used from a variety of suppliers (Northern graphite, Timcal, Qingdao Henglide Graphite, Asbury Graphite, Future Carbon and Sigma-Aldrich). The lateral 
dimensions of the graphite samples were measured by Zeiss Ultra Plus Field Emission SEM and software ImageJ was used measure flake length.

Specific surface area and surface energy measurements were carried out on a Surface Energy Analyser (SEA) IGC (Surface Measurement Systems, UK). All IGC samples were packed into silanised glass columns of internal diameter (ID) of $2 \mathrm{~mm}$ and length $30 \mathrm{~cm}$. Silanised glass wool was used to plug both ends of column containing to sample to prevent machine contamination. For Specific surface area measurement 15-20mg of sample were packed into a $2 \mathrm{~mm}$ ID column and preconditioned at $150{ }^{\circ} \mathrm{C}$ under Helium with flow rate 20 $\mathrm{mL} / \mathrm{min}$ to remove and surface moisture. The peak max method was used for the retention time. A series of increasing probe concentrations of Octane at $20{ }^{\circ} \mathrm{C}$ and flow rate $20 \mathrm{~mL} / \mathrm{min}$ were injected into the column to get an adsorption isotherm. Methane was used to calculate the column dead time. From this the BET equation is applied using the SMS Cirrus Plus software to get the samples specific surface area.

The surface energy of the samples was measured by packing columns with a mass that corresponds to a surface area of $0.5 \mathrm{mg} / \mathrm{m}^{2}$, this known from specific surface area measurement. The samples were preconditioned in the same way as previously described. Alkane probes (nonane, octane, heptane, hexane) were used to measure the dispersive surface energy. Coverage values ranging from 0.0075 up to a maximum of 0.4 were used to measure surface energy profiles of the samples. The peak centre of mass (COM) method was used to measure retention time. Surface energy measurements were performed at temperatures from $60-80{ }^{\circ} \mathrm{C}$. The Cirrus plus software was used for all calculations. Chromatogram elution times were measured using a flame ionising detector (FID). The accuracy of the reported $\gamma_{d}$ values depends heavily on the quality of the linear fit of the alkane line since the Dorris-Gray surface energy value depends on the square of the slope as show in equation 5. As a result we only used values where $\mathrm{R}^{2}>0.99$ for alkane line fits. The uncertainty in $\gamma_{d}$ measurements is explored in more detail by Ylä-Mäihäniemi et al ${ }^{42}$.

Raman spectroscopy was performed on the graphite samples using a Horiba Jobin Yvon LabRAM HR800 with $532 \mathrm{~nm}$ excitation laser in air under ambient conditions. The Raman emission was collected by $100 \times$ objective lens (N.A. $=0.8$, spot size $\sim 1 \mu \mathrm{m}$ ). To avoid sample heating we carried out all Raman experiments at $10 \%$ of maximum laser power $(<2 \mathrm{~mW})$. A mapping over a $20 \times 20 \mu \mathrm{m}^{2}$ sample area was performed in each case in $2 \mu \mathrm{m}$ steps. The spectra displayed are the baseline-corrected average of 120 individual spectra. 


\section{Simulations}

In order to calculate the surface energy profiles, we use the method of Jefferson et al. ${ }^{56}$ as modified by Smith et al. ${ }^{57}$. The reader is referred to these papers for a full description. This model assumes the surface can be divided into one or more populations of binding sites. When probe molecules are introduced, they bind to the highest energy sites following a Boltzman distribution until the appropriate coverage is achieved. Then the surface energy can be calculated from the population-weighted mean of the binding energy.

The binding site populations are represented by normal distributions of the type shown in equation 8. In general, the total distribution can be the sum over a number of such Gaussians, each representing a different population of site. The total distribution is the transformed from continuous to discrete with bin size $0.005 \mathrm{~mJ} / \mathrm{m}^{2}$ and truncated for energies $<0 \mathrm{~mJ} / \mathrm{m}^{2}$ to avoid introducing unphysical negative surface energies.

To account for the fact that probes preferentially adsorb to higher energy sites ${ }^{69}$, we use a Boltzmann distribution to calculate the relative occupancy of each site, shown in equation 12 $P_{i}=\frac{e^{-E_{i} / k T}}{\sum_{i} e^{-E_{i} / k T}}$

where $\mathrm{P}_{\mathrm{i}}$ is the number probability of a probe molecule adsorbing to site $i$, which has a probesite binding energy of $\mathrm{E}_{\mathrm{i}}$. This binding energy is taken to be equal to the Gibbs free energy of adsorption, $\Delta G_{\text {probe }}$ :

$$
E_{i}=\Delta G_{\text {probe }}=-a_{\text {probe }} 2 \sqrt{\gamma_{d} \gamma_{d}^{\text {probe }}}
$$

Where $a_{\text {probe }}$ is the area occupied by each probe and $\gamma_{d}^{\text {probe }}$ is the surface energy of the probe. Substituting equation 13 into equation 12 and multiplying by the total site distribution (generally a sum of discrete Gaussians distributions) gives the probability of a probe filling a site $i /$ fraction of injected probes at each site.. The surface energy at that coverage is then given by the mean of the surface energy contribution, weighted by the fraction of probe molecules at each energy (in each bin). However, it is known that this method gives values of surface energy which depend on the probe surface energy. This requires us to apply a correction procedure which is described in reference ${ }^{57}$. 


\section{ASSOCIATED CONTENT}

Supplemental Information

SEM images of each graphite sample with flake length distribution histograms, BET surface area equations, Raman spectra for each graphite sample used in the study, Raman $I_{D} / I_{G}$ as function of inverse flake length, simulated dispersive surface energy profiles, simulated dispersive surface energy profiles fitted to both standard and stretched/compressed exponentials, measured dispersive surface energy profiles for graphite samples in the study, perimeter to area ratio calculation, comparison of data to literature calculations. This material is available free of charge via the Internet at http://pubs.acs.org.

\section{ACKNOWLEDGEMENTS:}

This work was primarily supported by and SFI PYRA grant as well as the SFI-funded AMBER research centre $(\mathrm{SFI} / 12 / \mathrm{RC} / 2278)$ as part of the platform projects program. In addition, we acknowledge the European Union Seventh Framework Program under grant agreement n604391 Graphene Flagship. 



Figure 1: (A) SEM image of 9-Henglide graphite. (B) Close up SEM image of 5-Timcal flake which shows that the flakes are not monolithic but are clearly made of thinner sub-structure. (C) Plot of specific surface area versus graphite flake length. The dashed line represents the mean of $8.2 \mathrm{~m}^{2} / \mathrm{g}$. (D) Raman spectra of 2 graphite samples with the main bands (D, $G$ and $2 D)$ indicated. This shows the ratio of $D$ to $G$ band intensities to vary among samples, implying a variation of defect contents.
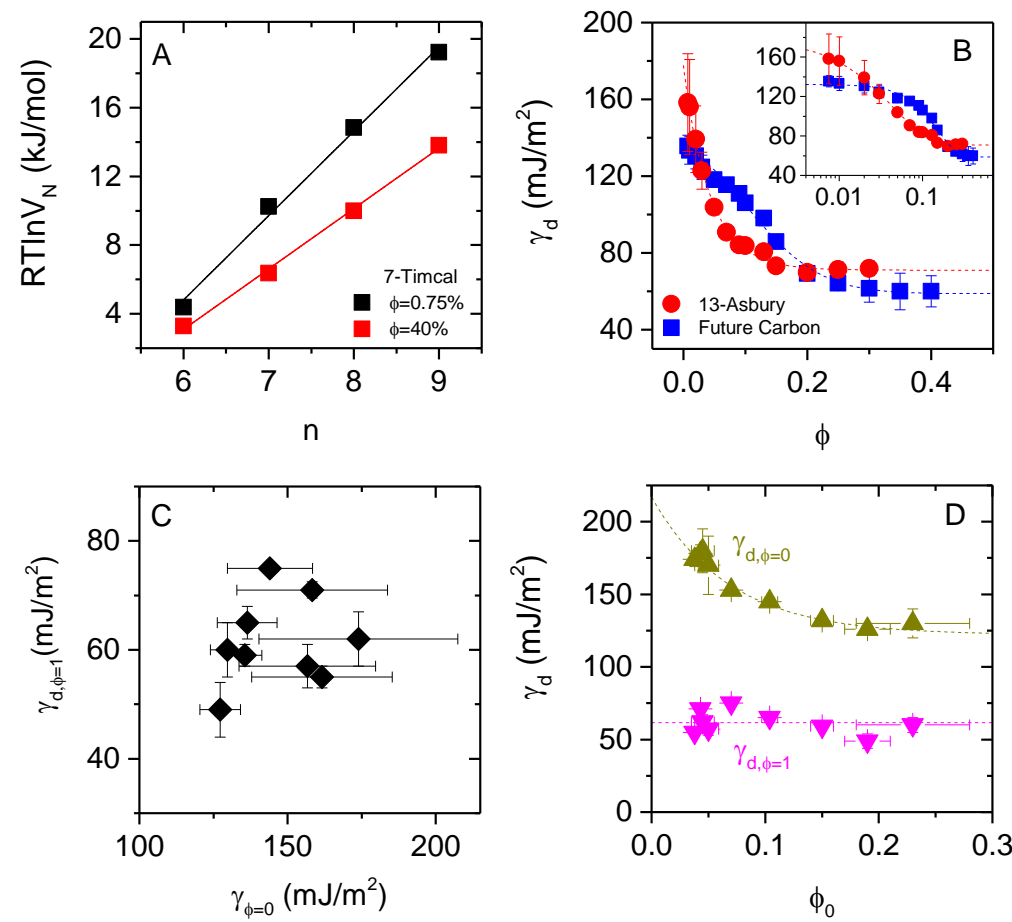

Figure 2: (A) The Gibbs free energy of adsorption $\left(R T \ln V_{N}\right)$ as a function of alkane number for two coverage values of the same sample (Timcal). The slope of the line is used for the 
calculation of $\gamma_{d}$ using the Dorris-Gray formula (equation 5). (B) $\gamma_{d}$ as a function of surface coverage $(\phi)$ for two of the graphite samples used in the study (Northern and 18-Asbury). $\gamma_{d}$ decreases with surface coverage until it reaches a plateau where it becomes coverage independent. The $\gamma_{d}$ value at the plateau is defined as $\gamma_{d, \phi=1}$, the full coverage dispersive surface energy value. The $\gamma_{d}$ profiles have been fitted to equation $7 b$ which is similar to the standard exponential decay typically used for heterogeneous solids. Inset: Semi-log plot. (C) $\gamma_{d, \phi=1}$ plotted against $\gamma_{d, \phi=0}$. This shows no correlation indicating that they are independent of each other. (D) Dispersive surface energy at both zero coverage $\left(\gamma_{d, \phi=0}\right)$ and full coverage ( $\left.\gamma_{d, \phi=1}\right)$ as a function of the decay constant $\left(\phi_{0}\right)$. The upper line shows an exponential decay while the lower line represents the mean value of $\gamma_{d, \phi=1}$.
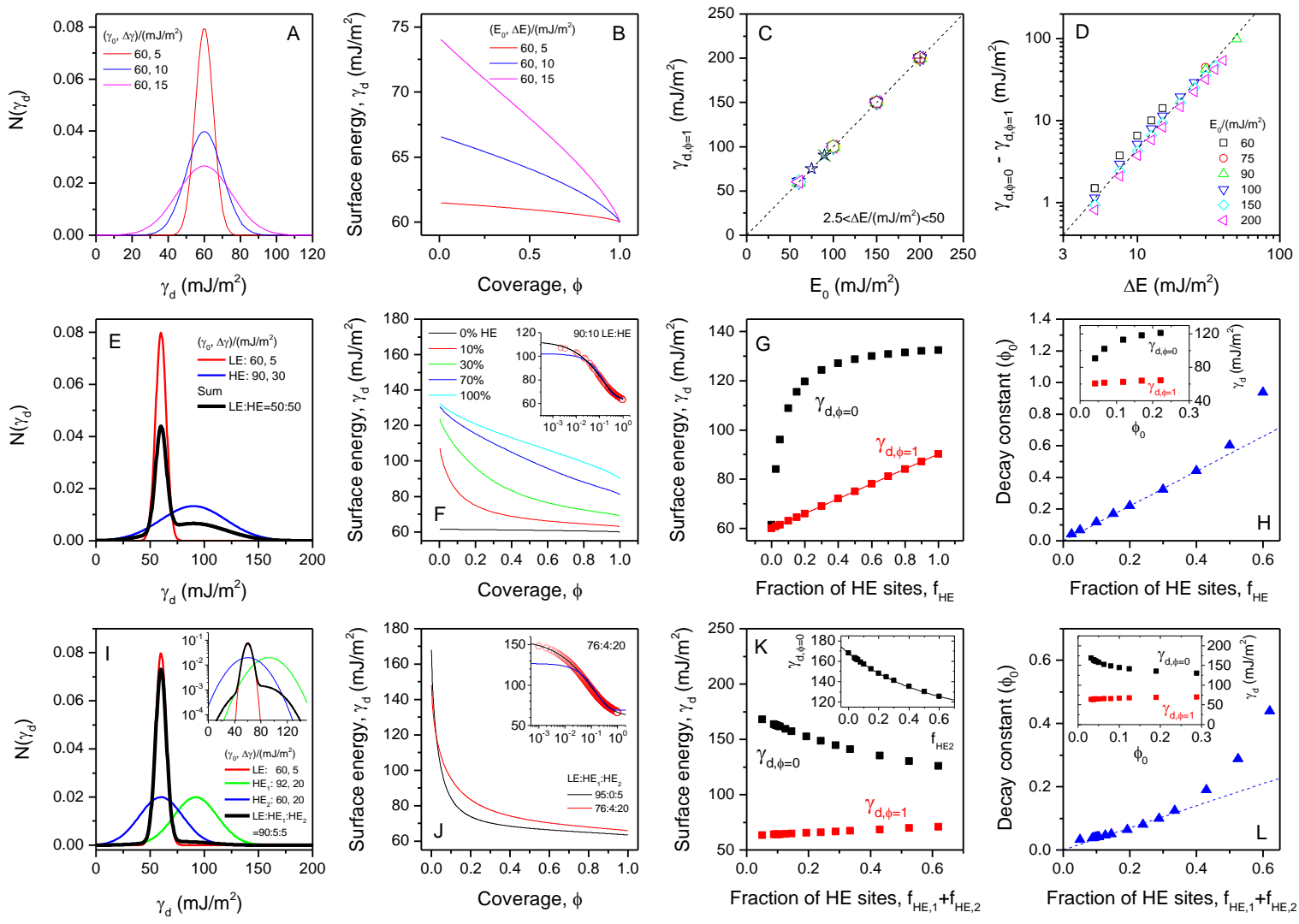

Figure 3: Simulation of surface energy vs. coverage data. A-D) Simulations involving one Gaussian distribution. A) Examples of Gaussian distributions of probe molecule-surface binding energies, expressed as energy per unit area (the distribution centres, $\gamma_{0}$ and widths, $\Delta \gamma$, are given in the panel). B) Simulated surface energy versus coverage profiles for the 
distributions shown in A). C) Calculated surface energy at full coverage ( $\phi=1)$ plotted versus distribution centre for a range of distributions with various widths. The different symbols represent different widths between 2.5 and $50 \mathrm{~mJ} / \mathrm{m}^{2}$. D) Difference between surface energy at zero coverage and that at full coverage plotted versus distribution width for various distributions with a range of centres. The line represents $\gamma_{d, \phi=0}-\gamma_{d, \phi=1}=0.045 \Delta \gamma^{2}$. E-H) Simulations involving two Gaussian distributions E) An example of a narrow low-energy and a broad high energy distribution with an equally weighted sum shown in black (the distribution centres, $\gamma_{0}$ and widths, $\Delta \gamma$, are given in the panel). F) Simulated surface energy versus coverage profiles for weighted combinations of the distributions shown in E. The weightings of the highenergy component are given in the panel. Inset: These distributions are not well fitted by exponential decays (blue line). Rather we find they are better described by stretched/compressed exponentials (black curve). G) Surface energy at zero- $(\phi=0)$ and fullcoverage $(\phi=1)$, calculated for weighted sums of the distributions in E) plotted versus the fraction of high-energy sites. $H$ ) Decay constants (found by fitting curves such as those in $F$ to stretched/compressed exponentials) plotted versus the fraction of high-energy sites. The dashed line shows that, so long as $<45 \%$ of sites are of high energy, the decay constant is roughly equal to the fraction of high-energy sites. Inset: Plot of zero- $(\phi=0)$ and full-coverage $(\phi=1)$ surface energies versus decay constant. Note that this data does not match that in figure 2D. I-L) Simulations involving three Gaussian distributions. I) An example of a narrow lowenergy and two broad high energy distributions with a weighted sum (90:5:5) shown in black (the distribution centres, $\gamma_{0}$ and widths, $\Delta \gamma$, are given in the panel). Inset: The same data plotted on a semi-log graph. J) Simulated surface energy versus coverage profiles for weighted combinations of the distributions shown in E. The weightings of the high-energy components are given in the panel. Inset: These distributions are not well fitted by exponential decays (blue line) but are better described by stretched/compressed exponentials (black curve). K) Surface energy at zero- $(\phi=0)$ and full-coverage $(\phi=1)$, calculated for weighted sums of the distributions in I) plotted versus the total fraction of high-energy sites. Inset: $\gamma_{d, \phi=0}$ plotted versus $f_{H E 2 .}$ The line represents an exponential decay. L) Decay constants (found by fitting curves such as those in $F$ to stretched/compressed exponentials) plotted versus the fraction of high-energy sites. The dashed line shows that, so long as <35\% of sites are of high energy, the decay constant is proportional to the total fraction of high-energy sites. Inset: Plot of zero- 
$(\phi=0)$ and full-coverage $(\phi=1)$ surface energies versus decay constant. Note that this data matches that in figure $2 D$ extremely well.
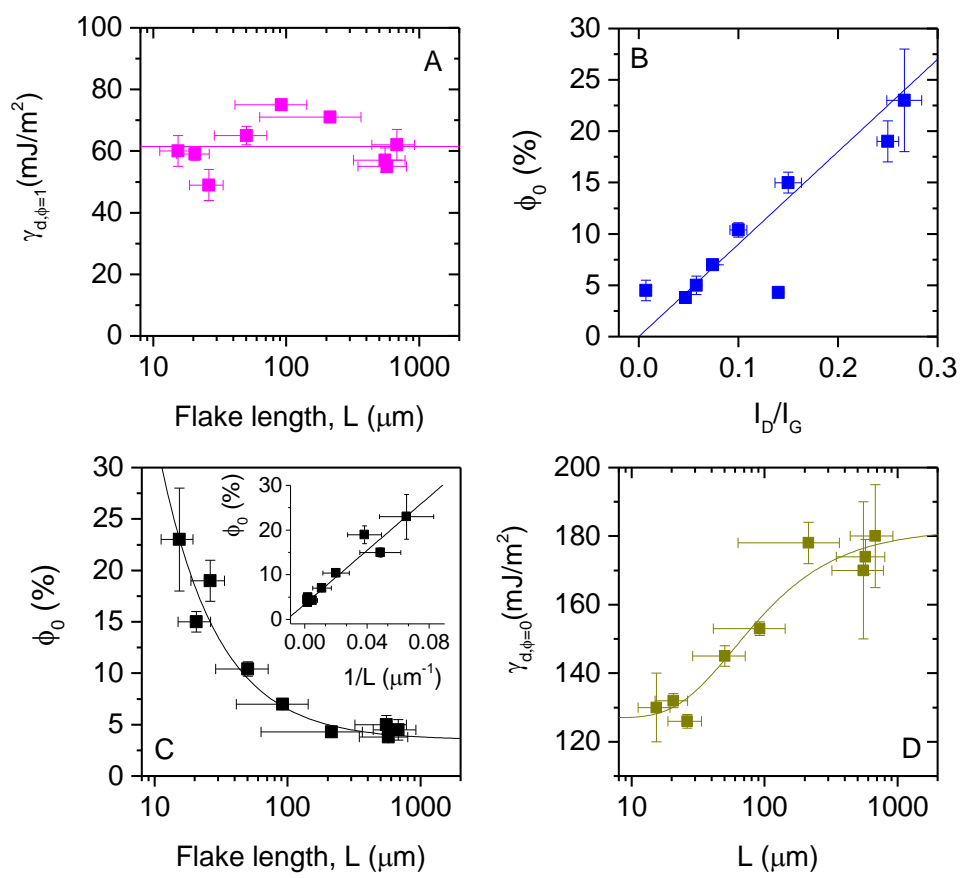

Figure 4: (A) $\gamma_{d, \phi=1}$ plotted as a function of mean flake length $(L)$ showing that $\gamma_{d, \phi=1}$ is size independent. (B) Decay constant, $\phi_{0}$, plotted as a function of $I_{D} / I_{G}$, the linear trend indicates that $\phi_{0}$ is related to the total amount of defects present in the sample. $(C) \phi_{0}$ plotted as a function of $L$ with the inset showing linear trend of $\phi_{0}$ with $1 / L$. Using equation $8 b$ this shows that $\phi_{0}$ gives information about the nature of defects present in the sample and is able to differentiate between edge and basal plane defects. (D) $\gamma_{d, \phi=0}$ plotted as a function of $L$ and fitted to an exponential function consistent with the simulated behaviour shown in figure $3 \mathrm{~K}$ inset. 


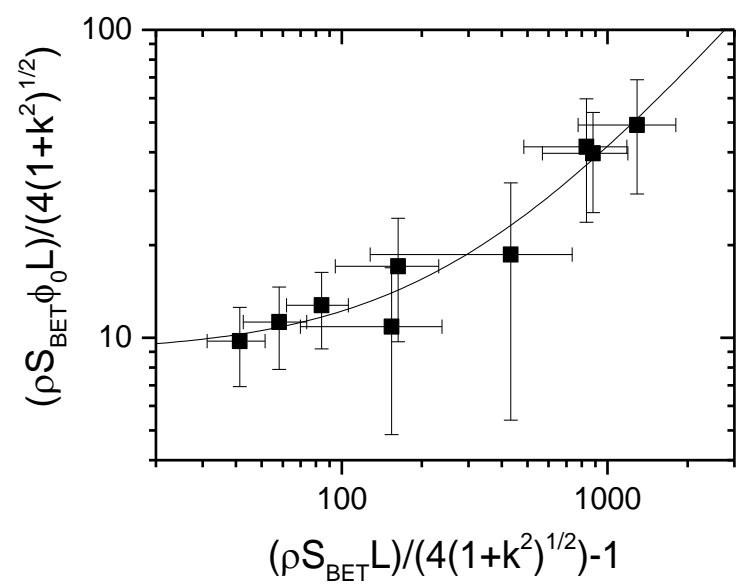

Figure 5: Combination of specific surface area and IGC data parameterised according to equation 11. The line is a linear fit.

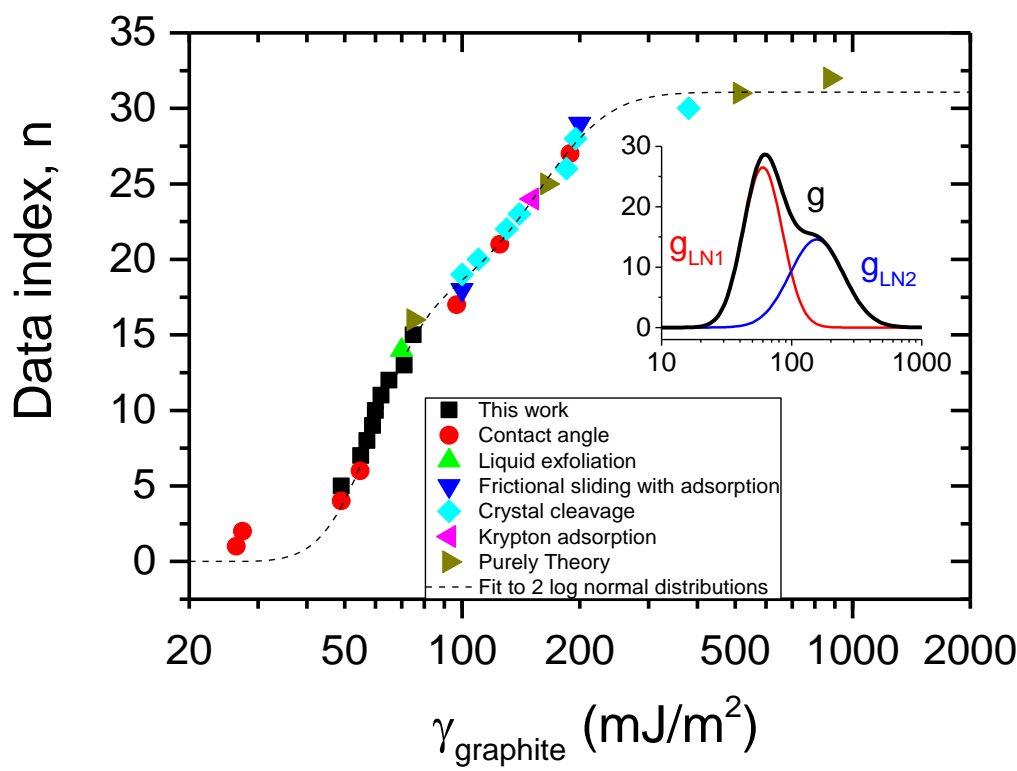

Figure 6: The measurements from this study compared to literature values in ascending order of $\gamma_{\text {graphite. }}$. The values are taken from contact angle, liquid exfoliation, frictional sliding with adsorption, crystal cleavage, Krypton adsorption and theoretical calculations. Our data is selfconsistent and also consistent with some contact angle and liquid exfoliation measurements. The dashed line is a fit to equation 11 which allows us to reconstruct the over-all graphite surface energy distribution, $g$, as shown in the inset. From the fit in the main panel we can infer that the over-all graphite surface energy distribution is the sum of two log-normal distributions $g_{L N 1}$ and $g_{L N 2}$. 


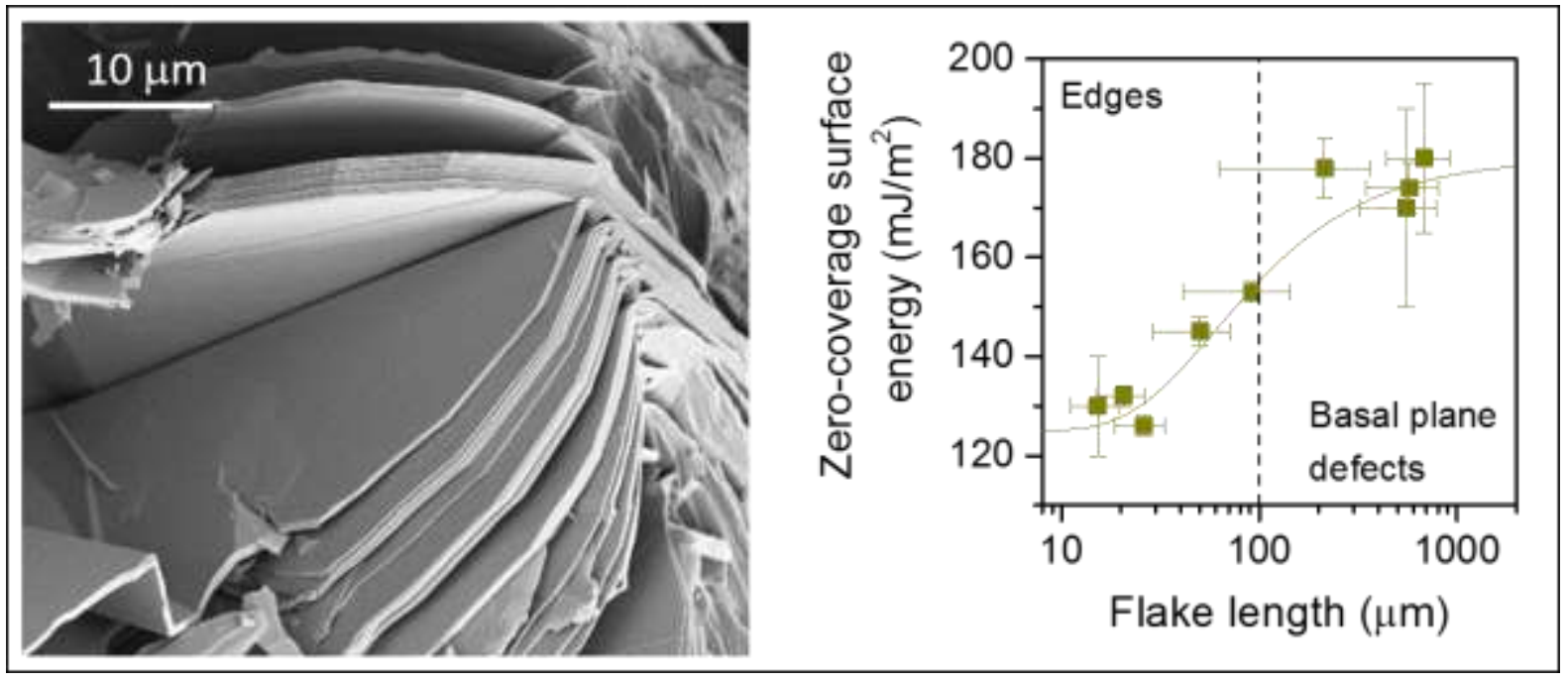

TOC fig

\section{References}

(1) Wallace, P. R. The Band Theory of Graphite. Phys. Rev. 1947, 71, 622-634.

(2) Hammer, G.; Drzal, L. Graphite Fiber Surface Analysis by X-Ray Photoelectron Spectroscopy and Polar/dispersive Free Energy Analysis. Appl. Surf. Sci. 1980, 4, 340-355.

(3) Bryant, P. J.; Gutshall, P. L.; Taylor, L. H. A Study of Mechanisms of Graphite Friction and Wear. Wear 1964, 7, 118-126.

(4) Thielmann, F.; Pearse, D. Determination of Surface Heterogeneity Profiles on Graphite by Finite Concentration Inverse Gas Chromatography. J. Chromatogr. A 2002, 969, 323-327.

(5) Novoselov, K. S.; Geim, A. K.; Morozov, S. V; Jiang, D.; Zhang, Y.; Dubonos, S. V; Grigorieva, I. V; Firsov, A. A. Electric Field Effect in Atomically Thin Carbon Films. Science 2004, 306, 666-669.

(6) Geim, A. K.; Novoselov, K. S. The Rise of Graphene. Nat. Mater. 2007, 6, 183-191.

(7) Mcallister, M. J.; Li, J.; Adamson, D. H.; Schniepp, H. C.; Abdala, A.; Liu, J.; Herreraalonso, O. M.; Milius, D. L.; Car, R.; Prud, R. K.; Aksay, I. a. Single Sheet Functionalized Graphene by Oxidation and Thermal Expansion of Graphite. Society 2007, 19, 4396-4404. 
(8) Hernandez, Y.; Nicolosi, V.; Lotya, M.; Blighe, F. M.; Sun, Z.; De, S.; McGovern, I. T.; Holland, B.; Byrne, M.; Gun'Ko, Y. K.; Boland, J. J.; Niraj, P.; Duesberg, G.;

Krishnamurthy, S.; Goodhue, R.; Hutchison, J.; Scardaci, V.; Ferrari, A. C.; Coleman, J. N. High-Yield Production of Graphene by Liquid-Phase Exfoliation of Graphite.

Nat. Nanotechnol. 2008, 3, 563-568.

(9) Paton, K. R.; Varrla, E.; Backes, C.; Smith, R. J.; Khan, U.; O’Neill, A.; Boland, C.; Lotya, M.; Istrate, O. M.; King, P.; Higgins, T.; Barwich, S.; May, P.; Puczkarski, P.; Ahmed, I.; Moebius, M.; Pettersson, H.; Long, E.; Coelho, J.; O’Brien, S. E.;

McGuire, E. K.; Sanchez, B. M.; Duesberg, G. S.; McEvoy, N.; Pennycook, T. J.; Downing, C.; Crossley, A.; Nicolosi, V.; Coleman, J. N. Scalable Production of Large Quantities of Defect-Free Few-Layer Graphene by Shear Exfoliation in Liquids. Nat. Mater. 2014, 13, 624-630.

(10) Grimsey, I. M.; Feeley, J. C.; York, P. Analysis of the Surface Energy of Pharmaceutical Powders by Inverse Gas Chromatography. J. Pharm. Sci. 2002, 91, 571-583.

(11) Ho, R.; Wilson, D. A.; Heng, J. Y. Y. Crystal Habits and the Variation in Surface Energy Heterogeneity. Cryst. Growth Des. 2009, 9, 4907-4911.

(12) Chehimi, M. M.; Abel, M.-L.; Perruchot, C.; Delamar, M.; Lascelles, S. F.; Armes, S. P. The Determination of the Surface Energy of Conducting Polymers by Inverse Gas Chromatography at Infinite Dilution. Synth. Met. 1999, 104, 51-59.

(13) Park, S. London Dispersive Component of the Surface Free Energy and Surface Enthalpy. J. Colloid Interface Sci. 1997, 188, 336-339.

(14) Munk, P. Study of Polymer-Polymer Interaction Coefficients in Polymer Blends Using Inverse Gas Chromatography. 1984, 17, 803-809.

(15) Ali, S. S. M.; Heng, J. Y. Y.; Nikolaev, A. A.; Waters, K. E. Introducing Inverse Gas Chromatography as a Method of Determining the Surface Heterogeneity of Minerals for Flotation. Powder Technol. 2013, 249, 373-377.

(16) Mukhopadhyay, P.; Schreiber, H. P. Aspects of Acid-Base Interactions and Use in IGC. Colloids Surfaces A Physicochem. Eng. Asp. 1995, 100, 47-71.

(17) Mohammadi-Jam, S.; Waters, K. E. Inverse Gas Chromatography Applications: A Review. Adv. Colloid Interface Sci. 2014, 212, 21-44.

(18) Voelkel, A.; Adamska, K.; Strzemiecka, B.; Batko, K. Determination of Hansen Solubility Parameters of Solid Materials by Inverse Gas-Solid Chromatography. Acta Chromatogr. 2008, 20, 1-14.

(19) Tyagi, O. S.; Deshpande, D. D. Inverse Gas Chromatography of Poly(n-Butyl Methacrylate): Effect of Flow Rate on Specific Retention Volume and Detection of Glass Transition Temperature. J. Appl. Polym. Sci. 1987, 34, 2377-2388. 
(20) James, A. T.; Martin, A. J. P. Gas-Liquid Partition Chromatography; the Separation and Micro-Estimation of Volatile Fatty Acids from Formic Acid to Dodecanoic Acid. Biochem. J. 1952, 50, 679-690.

(21) Ortmann, F.; Bechstedt, F.; Schmidt, W. G. Semiempirical van Der Waals Correction to the Density Functional Description of Solids and Molecular Structures. Phys. Rev. B - Condens. Matter Mater. Phys. 2006, 73, 1-10.

(22) Van Oss, C. J.; Good, R. J.; Chaudhury, M. K. Interfacial Lifshitz-van Der Waals and Polar Interactions in Macroscopic Systems. Chem. Rev. 1988, 88, 927-941.

(23) Schultz, J.; Lavielle, L.; Martin, C. The Role of the Interface in Carbon Fibre-Epoxy Composites. J. Adhes. 1987, 23, 45-60.

(24) Dorris, G. M.; Gray, D. G. Adsorption of N-Alkanes at Zero Surface Coverage on Cellulose Paper and Wood Fibers. J. Colloid Interface Sci. 1980, 77, 353-362.

(25) Shi, B.; Wang, Y.; Jia, L. Comparison of Dorris-Gray and Schultz Methods for the Calculation of Surface Dispersive Free Energy by Inverse Gas Chromatography. $J$. Chromatogr. A 2011, 1218, 860-862.

(26) Fowkes, F. M. Attractive Forces at Interfaces. Ind. Eng. Chem. 1964, 56, 40-52.

(27) Dong, S.; Brendlé, M.; Donnet, J. B. Study of Solid Surface Polarity by Inverse Gas Chromatography at Infinite Dilution. Chromatographia 1989, 28, 469-472.

(28) Papirer, E.; Li, S.; Balard, H.; Jagiello, J. Surface Energy and Adsorption Energy Distribution Measurements on Some Carbon Blacks. Carbon 1991, 29, 1135-1143.

(29) Ho, R.; Heng, J. Y. Y. A Review of Inverse Gas Chromatography and Its Development as a Tool to Characterize Anisotropic Surface Properties of Pharmaceutical Solids. KONA Powder Part. J. 2013, 30, 164-180.

(30) Huson, M. G.; Church, J. S.; Kafi, A. A.; Woodhead, A. L.; Khoo, J.; Kiran, M. S. R. N.; Bradby, J. E.; Fox, B. L. Heterogeneity of Carbon Fibre. Carbon 2014, 68, 240249.

(31) Kondor, A.; Quellet, C.; Dallos, A. Surface Characterization of Standard Cotton Fibres and Determination of Adsorption Isotherms of Fragrances by IGC. Surf. Interface Anal. 2015, 47, 1040-1050.

(32) Ferrari, A. C. Raman Spectroscopy of Graphene and Graphite: Disorder, ElectronPhonon Coupling, Doping and Nonadiabatic Effects. Solid State Commun. 2007, 143, 47-57.

(33) Ferrari, A.; Robertson, J. Interpretation of Raman Spectra of Disordered and Amorphous Carbon. Phys. Rev. B 2000, 61, 14095-14107.

(34) Dresselhaus, M. S.; Dresselhaus, G.; Saito, R.; Jorio, a. Raman Spectroscopy of Carbon Nanotubes. Phys. Rep. 2005, 409, 47-99. 
(35) Pimenta, M. a; Dresselhaus, G.; Dresselhaus, M. S.; Cançado, L. G.; Jorio, a; Saito, R. Studying Disorder in Graphite-Based Systems by Raman Spectroscopy. Phys. Chem. Chem. Phys. 2007, 9, 1276-1291.

(36) Eckmann, A.; Felten, A.; Mishchenko, A.; Britnell, L.; Krupke, R.; Novoselov, K. S.; Casiraghi, C. Probing the Nature of Defects in Graphene by Raman Spectroscopy. Nano Lett. 2012, 12, 3925-3930.

(37) Cançado, L. G.; Jorio, a.; Ferreira, E. H. M.; Stavale, F.; Achete, C. a.; Capaz, R. B.; Moutinho, M. V. O.; Lombardo, a.; Kulmala, T. S.; Ferrari, a. C. Quantifying Defects in Graphene via Raman Spectroscopy at Different Excitation Energies. Nano Lett. 2011, 11, 3190-3196.

(38) Lespade, P.; Marchand, A.; Couzi, M.; Cruege, F. Caracterisation de Materiaux Carbones Par Microspectrometrie Raman. Carbon 1984, 22, 375-385.

(39) Cançado, L. G.; Pimenta, M. a.; Neves, B. R. a; Dantas, M. S. S.; Jorio, a. Influence of the Atomic Structure on the Raman Spectra of Graphite Edges. Phys. Rev. Lett. 2004, 93, 5-8.

(40) Beams, R.; Cançado, L. G.; Novotny, L. Raman Characterization of Defects and Dopants in Graphene. J. Phys. Condens. Matter 2015, 27, 083002.

(41) Mohammadi-Jam, S.; Burnett, D. J.; Waters, K. E. Surface Energy of Minerals Applications to Flotation. Miner. Eng. 2014, 66-68, 112-118.

(42) Ylä-Mäihäniemi, P. P.; Heng, J. Y. Y.; Thielmann, F.; Williams, D. R. Inverse Gas Chromatographic Method for Measuring the Dispersive Surface Energy Distribution for Particulates. Langmuir 2008, 24, 9551-9557.

(43) Gamble, J. F.; Davé, R. N.; Kiang, S.; Leane, M. M.; Tobyn, M.; Wang, S. S. Y. Investigating the Applicability of Inverse Gas Chromatography to Binary Powdered Systems: An Application of Surface Heterogeneity Profiles to Understanding Preferential Probe-Surface Interactions. Int. J. Pharm. 2013, 445, 39-46.

(44) Lapčík, L.; Lapčíková, B.; Otyepková, E.; Otyepka, M.; Vlček, J.; Buňka, F.; Salek, R. N. Surface Energy Analysis (SEA) and Rheology of Powder Milk Dairy Products. Food Chem. 2015, 174, 25-30.

(45) Ferguson, A.; Khan, U.; Walsh, M.; Lee, K.-Y.; Bismarck, A.; Shaffer, M. S. P.; Coleman, J. N.; Bergin, S. D. Understanding the Dispersion and Assembly of Bacterial Cellulose in Organic Solvents. Biomacromolecules 2016, 17, 1845-1853.

(46) Yao, Z.; Ge, L.; Yang, W.; Xia, M.; Ji, X.; Jin, M.; Tang, J.; Dienstmaier, J. Finite Dilution Inverse Gas Chromatography as a Versatile Tool To Determine the Surface Properties of Biofillers for Plastic Composite Applications. Anal. Chem. 2015, 87, 6724-6729. 
(47) Balard, H.; Maafa, D.; Santini, A.; Donnet, J. B. Study by Inverse Gas Chromatography of the Surface Properties of Milled Graphites. J. Chromatogr. A 2008, 1198-1199, 173-180.

(48) Papirer, E.; Brendle, E.; Ozil, F.; Balard, H. Comparison of the Surface Properties of Graphite, Carbon Black and Fullerene Samples, Measured by Inverse Gas Chromatography. Carbon 1999, 37, 1265-1274.

(49) Donnet, J. B.; Park, S. J.; Brendle, M. The Effect of Microwave Plasma Treatment on the Surface Energy of Graphite as Measured by Inverse Gas Chromatography. Carbon 1992, 30, 263-268.

(50) Wang, S.; Zhang, Y.; Abidi, N.; Cabrales, L. Wettability and Surface Free Energy of Graphene Films. Langmuir 2009, 25, 11078-11081.

(51) Hernandez, Y.; Nicolosi, V.; Lotya, M.; Blighe, F. M.; Sun, Z.; De, S.; McGovern, I. T.; Holland, B.; Byrne, M.; Gun'Ko, Y. K.; Boland, J. J.; Niraj, P.; Duesberg, G.; Krishnamurthy, S.; Goodhue, R.; Hutchison, J.; Scardaci, V.; Ferrari, A. C.; Coleman, J. N. High-Yield Production of Graphene by Liquid-Phase Exfoliation of Graphite. Nat. Nanotechnol. 2008, 3, 563-568.

(52) Good, R. J. Contact Angle, Wetting, and Adhesion: A Critical Review. J. Adhes. Sci. Technol. 1992, 6, 1269-1302.

(53) Lotya, M.; King, P. J.; Khan, U.; De, S.; Coleman, J. N. High-Concentration, Surfactant-Stabilized Graphene Dispersions. ACS Nano 2010, 4, 3155-3162.

(54) Li, Z.; Wang, Y.; Kozbial, A.; Shenoy, G.; Zhou, F.; McGinley, R.; Ireland, P.; Morganstein, B.; Kunkel, A.; Surwade, S. P.; Li, L.; Liu, H. Effect of Airborne Contaminants on the Wettability of Supported Graphene and Graphite. Nat. Mater. 2013, 12, 925-931.

(55) Ho, R.; Naderi, M.; Heng, J. Y. Y.; Williams, D. R.; Thielmann, F.; Bouza, P.; Keith, A. R.; Thiele, G.; Burnett, D. J. Effect of Milling on Particle Shape and Surface Energy Heterogeneity of Needle-Shaped Crystals. Pharm. Res. 2012, 29, 2806-2816.

(56) Jefferson, A. E.; Williams, D. R.; Heng, J. Y. Y. Computing the Surface Energy Distributions of Heterogeneous Crystalline Powders. J. Adhes. Sci. Technol. 2012, 25, 339-355.

(57) Smith, R. R.; Williams, D. R.; Burnett, D. J.; Heng, J. Y. Y. A New Method To Determine Dispersive Surface Energy Site Distributions by Inverse Gas Chromatography. Langmuir 2014, 30, 8029-8035.

(58) Barreiro, A.; Lazzeri, M.; Moser, J.; Mauri, F.; Bachtold, A. Transport Properties of Graphene in the High-Current Limit. Phys. Rev. Lett. 2009, 103, 2-5.

(59) Raj, R.; Maroo, S. C.; Wang, E. N. Wettability of Graphene. Nano Lett. 2013, 13, $1509-1515$. 
(60) Staszczuk, P. Determination of Water Film Pressure and Surface Free Energy on Graphite. Mater. Chem. Phys. 1986, 14, 279-287.

(61) Schrader, M. E. Ultrahigh-Vacuum Techniques in the Measurement of Contact Angles. 5. LEED Study of the Effect of Structure on the Wettability of Graphite. J. Phys. Chem. 1980, 84, 2774-2779.

(62) Zaidi, H.; Robert, F.; Paulmier, D. Influence of Adsorbed Gases on the Surface Energy of Graphite: Consequences on the Friction Behaviour. Thin Solid Films 1995, 264, 4651 .

(63) Abrahamson, J. The Surface Energies of Graphite. Carbon 1973, 11, 337-362.

(64) Wang, W.; Dai, S.; Li, X.; Yang, J.; Srolovitz, D. J.; Zheng, Q. Measurement of the Cleavage Energy of Graphite. Nat. Commun. 2015, 6, 7853.

(65) Putnam, F. A.; Fort, T. Physical Adsorption on Patchwise Heterogeneous Surfaces .1. Heterogeneity, 2-Dimensional Phase-Transitions, and Spreading Pressure of KryptonGraphitized Carbon-Black System near 100 K. J. Phys. Chem. 1975, 79, 459-467.

(66) Ooi, N.; Rairkar, A.; Adams, J. B. Density Functional Study of Graphite Bulk and Surface Properties. Carbon 2006, 44, 231-242.

(67) Girifalco, L. A.; Lad, R. A. Energy of Cohesion, Compressibility, and the Potential Energy Functions of the Graphite System. J. Chem. Phys. 1956, 25, 693-697.

(68) Brennan, R. O. The Interlayer Binding in Graphite. J. Chem. Phys. 1952, 20, 40-48.

(69) Newell, H. E.; Buckton, G. Inverse Gas Chromatography: Investigating Whether the Technique Preferentially Probes High Energy Sites for Mixtures of Crystalline and Amorphous Lactose. Pharm. Res. 2004, 21, 1440-1444. 